\title{
The First Case of Benign Solitary Schwannoma Arising in the Rectus Abdominis Muscle
}

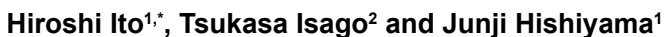

${ }^{1}$ Tokyo Women's Medical University, Shinjuku-ku, Tokyo, Japan

${ }^{2}$ Department of Plastic and Reconstructive Surgery, Tokyo Women's Medical University Medical Center East, Japan

\begin{abstract}
We presented a rare case of schwannoma arising from the rectus abdominis muscle in a 26 -year-old woman. She had referred to our department with a complaint of painless swelling on the right side of the umbilical region. The mass was about $4 \mathrm{~cm}$ in diameter, flat, elastic, and immobile. Ultrasonographic examination revealed a $37 \times 30 \mathrm{~mm}$ cystic lesion in the right rectus abdominis muscle and showed a heterogeneous low-echo area within the cyst. Magnetic resonance (MR) imaging examination of the abdomen showed a low-intensity in T1-weighted imaging, and T2-weighted images indicated niveau formation of a high-intensity area and a low-intensity area. With these findings, the patient was scheduled for surgery. Surgical specimen was $3 \times 2.5 \mathrm{~cm}$ surface-flat cystic tumor containing bloody fluid, and pathological diagnosis was schwannoma associated with cystic degeneration. This is the first case, to our knowledge, of schwannoma arising in the rectus abdominis muscle.
\end{abstract}

Keywords: Schwannoma; Neurinoma; Rectus abdominis muscle

\section{Introduction}

Schwannomas are solitary cystic benign tumors deriving from schwann cells in the peripheral nerves [1]. The tumors can develop anywhere the peripheral nerves are present, but commonly arise in the head and neck, spinal cord, and extremities [2]. We encountered a rare case in which a schwannoma arose in the rectus abdominis muscle of the abdomen, and herein report the case.

\section{Case Report}

A 26-year-old woman presented with a 1-year history of mass in her abdomen. Her past history and family history were unremarkable. She consulted a local physician. Ultrasonographic and computed tomographic (CT) images of the abdomen revealed a cystic lesion in the right rectus abdominis muscle. Paracentesis yielded clear yellow fluid. Cytologic diagnosis of this fluid was class II, which meant atypical cytology but no evidence of malignancy. She was referred to our department for thorough examination and treatment. Physical examination was significant for a flat, elastic, and immobile mass, measuring $4 \mathrm{~cm}$ in diameter, located in the right umbilical region. She had no spontaneous pain but tenderness. Ultrasonographic examination revealed a $37 \times 30 \mathrm{~mm}$ cystic lesion in the right rectus abdominis muscle and showed a heterogeneous low-echo area within the cyst (Figure 1). The tumor remained within the muscle. It displaced the fascia partially but did not appear to have extended beyond the fascia nor infiltrated the abdominal cavity. Magnetic resonance (MR) imaging examination of the abdomen revealed a mass legion measuring $3 \mathrm{~cm}$ in diameter in the right rectus abdominis muscle. T1-weighted MR images showed a low-intensity area, and T2-weighted images indicated niveau formation of a high-intensity area and a low-intensity area (Figure 2A and 2B).

On the basis of the clinical course and the diagnostic imaging, we suspected hemangioma of the abdomen, haematoma of the rectus abdominis muscle, and an abdominal wall endometriosis, and performed surgery on the patient under general anesthesia.

An incision was made immediately above the tumor, and the anterior rectus sheath was exposed. An incision made in the sheath showed that the tumor arose in the rectus abdominis muscle (Figure 3 ). When the muscle was opened by blunt dissection, there were no adhesions between the tumor and the surrounding tissues. The tumor could easily be removed from the posterior rectus sheath, and the tumor and its capsule were resected as en block. There were no grossly apparent nerve fibers. Surgical specimen was $3 \times 2.5 \mathrm{~cm}$ cystic tumor containing bloody fluid (Figure 4).

Histopathological findings showed that the wall of the cyst was composed of thick collagen cells, which were lined with cells containing oval to compact spindle nuclei and in part with spindle cells containing twisted nuclei. The tumor was chiefly composed of loose myxoid

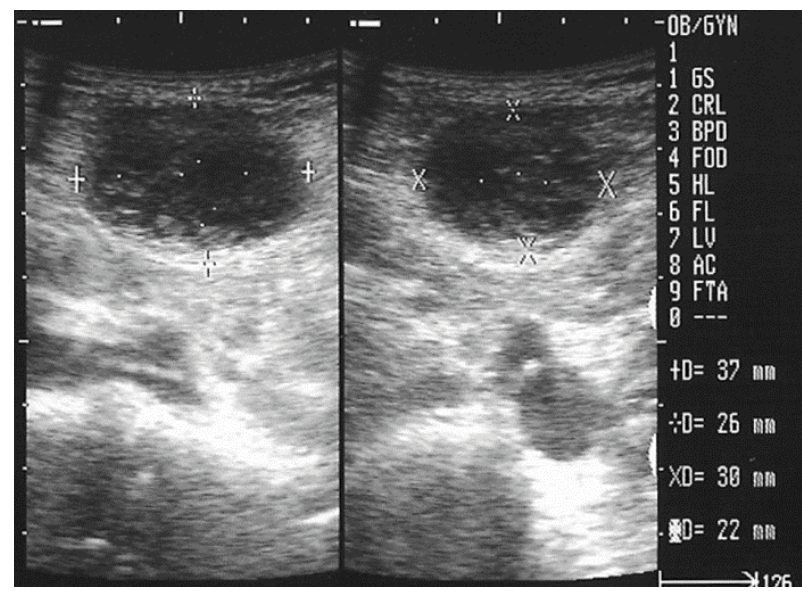

Figure 1: Ultrasonographic examination revealed a cystic lesion in the right rectus abdominis muscle.

*Corresponding author: Hiroshi lto, MD, PhD, Associate Professor, Tokyo Women's Medical University Plastic and Reconstructive Surgery 8-1, Kawada-cho, Shinjukuku, Tokyo 162-8666, Japan, Tel: 81-3-3353-8111; E-mail: ihiroshi@coffee.ocn.ne.jp

Received August 12, 2015; Accepted September 14, 2015; Published October 21, 2015

Citation: Ito $\mathrm{H}$, Isago T, Hishiyama J (2015) The First Case of Benign Solitary Schwannoma Arising in the Rectus Abdominis Muscle. Surgery Curr Res 5: 248. doi:10.4172/2161-1076.1000248

Copyright: (c) 2015 Ito $\mathrm{H}$, et al. This is an open-access article distributed under the terms of the Creative Commons Attribution License, which permits unrestricted use, distribution, and reproduction in any medium, provided the original author and source are credited. 
Citation: Ito H, Isago T, Hishiyama J (2015) The First Case of Benign Solitary Schwannoma Arising in the Rectus Abdominis Muscle. Surgery Curr Res 5: 248. doi:10.4172/2161-1076.1000248

Page 2 of 3

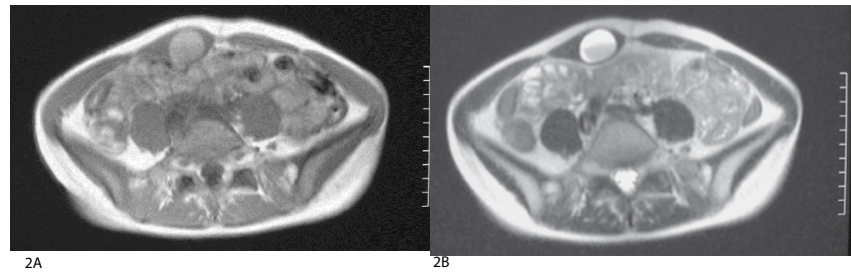

Figure 2A: T1-weighted MR images showed a low-intensity area. B: T2 weighted MR images indicated niveau formation of a high-intensity area and low-intensity area.

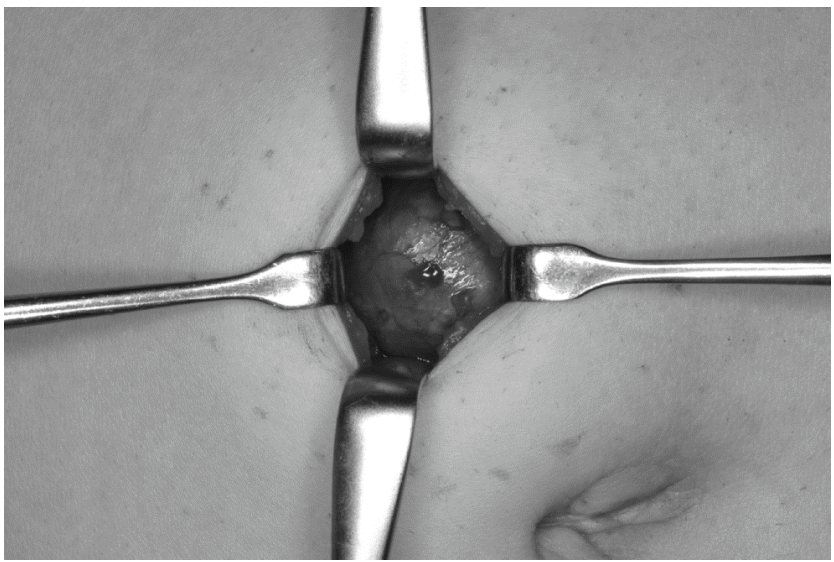

Figure 3: Intraoperative findings- An incision made in the anterior rectus sheath showed that the tumor arose in the rectus abdominis muscle.

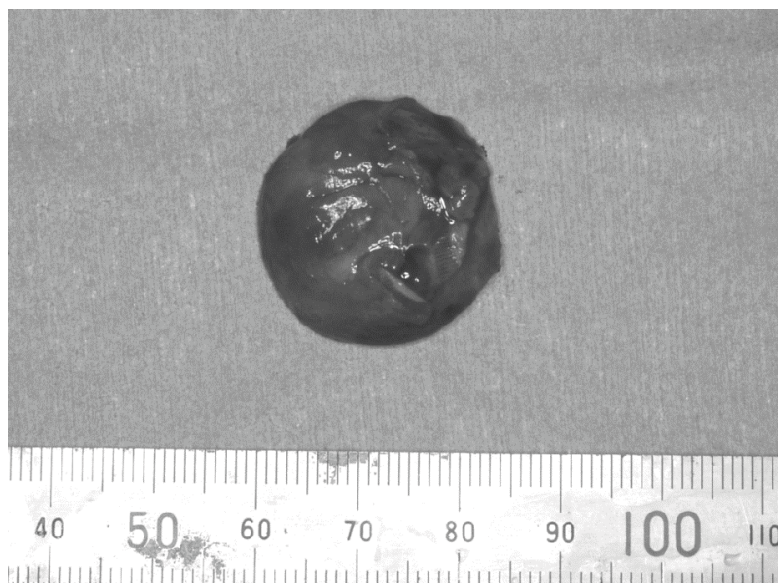

Figure 4: Surgical specimen was $3 \times 2.5 \mathrm{~cm}$ surface-flat cystic tumor containing bloody fluid.

component (Antoni B areas) and, partly, of highly ordered cellular component (Antoni A areas), without palisading (Figure 5). Within the cyst, bleeding and hemosiderosis were present. Since the tumor was positive for S-100 protein on immunostaining, a diagnosis of schwannoma associated with cystic degeneration was made. There were no signs of malignancy.

As of one year postoperatively, she has had an uneventful postoperative course. Clinical findings and CT images of the abdomen showed no evidence of recurrent tumors (Figure 6).

\section{Discussion}

Major diseases associated with tumors of the abdominal wall include abdominal hernia, urachal cystoma, haematoma of the rectus abdominis muscle [3], chronic inflammatory tumors (Schloffer's tumor) [4], abdominal wall endometriosis [5], lipoma, fibroma, which are all benign tumors, and disseminated metastases of intraperitoneal-organ tumors to the abdominal wall. Rare tumors, including malignant fibrous histiocytoma [6], synovial sarcoma [7], and hemangiopericytoma, were also reported. However, since there is no report of schwannomas arising in the rectus abdominis muscle in the English-language literature. We consider the tumor in the present case very rare.

In 1910, Verocay [8] reported that schwannoma is a neurogenic tumor derived from schwann cells of the myelinated nerve. Many synonymes of schwannoma, including neurinoma, neurilemmoma, etc. are being used. Das Gupta et al. [2] reported that $44.9 \%$ of benign schwannomas, such as acoustic schwannoma, frequently arise in the head and neck, and most of the residual benign schwannomas arise in the spinal cord and the extremities; and only approximately $4 \%$ arise in the retroperitonium. This suggests that schwannoma arising in the rectus abdominis muscle in the abdomen is very rare.

No clinical symptoms are characteristic of schwannoma, and the chief complaint is concerning the symptoms associated with displacement of the surrounding organs by slowly growing tumors. In the present case, the tumor arose in the rectus abdominis muscle,

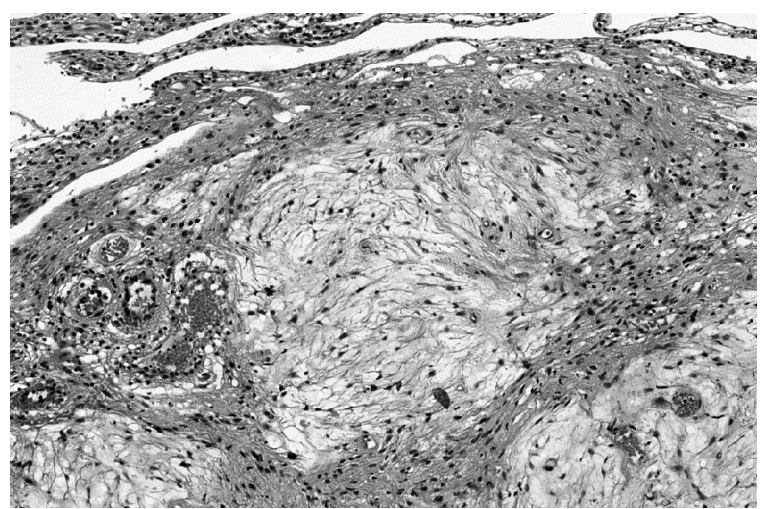

Figure 5: Postoperative histopathology: the tumor was chiefly composed of loose myxoid component (Antoni B areas) and, partly, of highly ordered cellular component (Antoni A areas), without palisading.

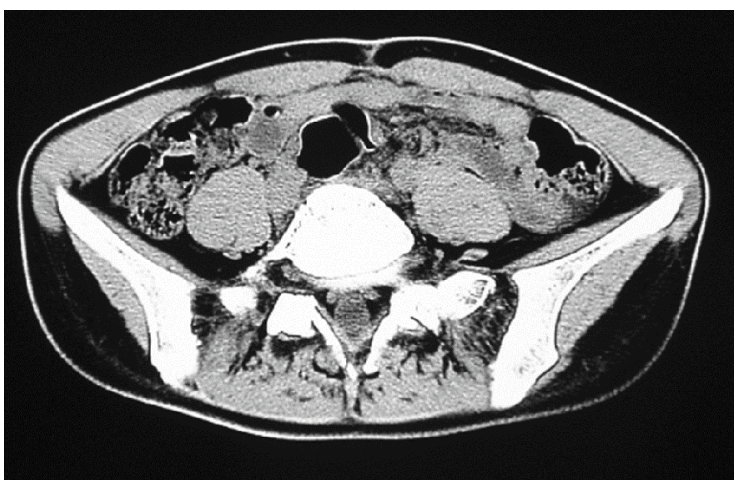

Figure 6: Postoperative CT findings showed no evidence of recurrence of tumor. 
where the intercostal nerves usually run. Therefore, we thought of the possibility that the tumor arose in the intercostal nerve in the rectus abdominis muscle. However, we could not determine whether the tumor arose in the intercostal nerves because there were no clear nerve fibers detected on intraoperative examination and no postoperative negative symptoms.

Histologically, the hallmark of a schwannoma is the pattern of alternating Antoni A and B areas. The relative amounts of these two components vary, and they may blend imperceptibly or change abruptly. Antoni A areas are composed of compact spindle cells that usually have twisted nuclei, indistinct cytoplasmic borders, and occasionally clear intranuclear vacuoles [1]. In highly differentiated Antoni A areas there may be nuclear palisading, whorling of the cells, and Verocay bodies. S-100 protein, an acidic protein common to supporting cells of the central and peripheral nervous system, can be demonstrated in schwannomas, particularly in the Antoni A areas [1]. Antoni B areas are far less orderly and less cellular. The spindle or oval cells are arranged haphazardly in the loosely textured matrix, which is punctuated by microcystic change, inflammatory cells, and delicate collagen fibers [1]. On occasion, schwannomas develop cystic spaces lined by Schwann cells that assume a round or epithelioid appearance. This change may be confused with true epithelial differentiation [1].

Ultrasonographic examination visualizes a schwannoma as a well-defined circular low-echo area. Echoes inside the tumor are heterogeneous, occasionally indicating a mixture pattern, which contains the echo pattern of a cyst. CT images show a well-defined circular multilobular tumor and occasionally visualize the inside of the tumor [9]. T1-weighted MR images show an almost homogeneous low-intensity area. T2-weighted images show a mixture of a low-intensity area and a high-intensity area in the Antoni A region and a homogeneous high-intensity area in the Antoni B region, according to the histological type [9].

The diagnostic imaging of the present tumor provided no characteristic findings of the disease. Therefore, diagnostic imaging is not sufficient to make a diagnosis, and pathologic evaluation is required. In the present case, cystic degeneration and intratumoral bleeding due to paracentesis and aspiration performed by another physician resulted in atypical MR images, which indicated niveau formation in the cyst, making the preoperative diagnosis difficult. With reference to the mechanism of the development of cystic schwannoma, such as the tumor in the present case, Enzynger et al. [1] thought that Antoni A areas are degenerated to become Antoni B areas. Therefore, recurrent tumors are less likely in schwannomas that underwent cystic degeneration of severe cellular degeneration and resultant scanty cellular constituents.

Radical excision of the tumor and its capsule is a rule of the treatment, and the outcomes are generally favorable. However, since there are reports of deaths from recurrent tumors and malignantly transformed tumors [10], we should carefully resect the capsule together with the tumor. In the present case, radical resection of the tumor and its capsule could be performed, and the likelihood of recurrence and metastasis appears to be very low. Nevertheless, we consider regular follow-up of the patient necessary.

\section{References}

1. Enzinger F, Weiss S (2001) Soft tissue tumors. (4th edn.) St. Louis: Mosby.

2. Das Gupta T, Brasfield R, Strong E, Hajdu SI (1969) Benign solitary Schwannomas (neurilemomas). Cancer 24: 355-366.

3. Gulamhuseinwala N, Webb P. (2005) Mushroom cloud appearance of hemorrhage into the superficial anterior. Clin Anat 18:141-145.

4. Gurlich R, Maruna P, Peskova M, Dorazilová V, Horejs J (2003) Severe late complication of appendicitis: Case report. Sb Lek 104: 139-143.

5. Esinler I, Guven S, Akyol D, Guvendag Guven ES, Taskiran C, et al. (2004) Abdominal wall endometriosis without previous surgery. J Obstet Gynaecol 24: 931.

6. Hartigan C, Holloway B (2005) Case report: MR imaging features of endometriosis at the umbilicus. Br J Radiol 78: 755-757.

7. Kakizoe E, Kakizoe F, Kakizoe K, Okunishi H (2002) Cutaneous malignant fibrous histiocytoma with the characteristics of different variants. Cutis 69: 211 214

8. Verocay J (1910) Zur kenntnis der 'Neurofibrome'. Beitr Path Anat 48: 1-60.

9. Rha S, Byun J, Jung S, Chun HJ, Lee HG, et al. (2003) Neurogenic tumors in the abdomen: tumor types and imaging characteristics. Radiographics 23 $29-43$

10. Das Gupta T, Brasfield R (1970) Solitary malignant schwannoma. Ann Surg 171: 419-428. 